\title{
Viscous Limits for strong shocks of one-dimensional systems of conservation laws
}

\section{Frédéric Rousset}

\begin{abstract}
We consider a piecewise smooth solution of a one-dimensional hyperbolic system of conservation laws with a single noncharacteristic Lax shock. We show that it is a zero dissipation limit assuming that there exist linearly stable viscous profiles associated with the discontinuities. In particular, following the approach of [7], we replace the smallness condition obtained by energy methods in [6] by a weaker spectra. The complete proofs can be found in [14].
\end{abstract}

\section{Introduction}

Consider a one-dimensional system of conservation laws

$$
u_{t}+f(u)_{x}=0
$$

with a smooth flux $f: \mathbb{R}^{n} \rightarrow \mathbb{R}^{n}$. We assume that (1) is hyperbolic, there exist smooth matrices $P(u), D(u)$ such that $P(u)^{-1} f^{\prime}(u) P(u)=D(u)$ where $D(u)$ is a diagonal matrix. The eigenvalues of $D$ will be denoted by $\lambda_{1}(u), \cdots, \lambda_{n}(u)$. We consider a piecewise smooth solution $u$ which is a distributional solution of (1) in the domain $\mathbb{R} \times\left[0, T^{*}\right]$ with a single shock, that is to say that $u(x, t)$ is smooth at any point $(x, t), x \neq s(t)$ where $x=s(t)$ is a smooth curve in the $(x, t)$ plane. Moreover the limits

$$
\begin{aligned}
& \partial_{x}^{k} u^{-}(t):=\partial_{x}^{k} u(s(t)-0, t)=\lim _{x \rightarrow s(t)^{-}} \partial_{x}^{k} u(x, t), \\
& \partial_{x}^{k} u^{+}(t):=\partial_{x}^{k} u(s(t)+0, t)=\lim _{x \rightarrow s(t)^{+}} \partial_{x}^{k} u(x, t),
\end{aligned}
$$

exist. We also assume that the shock is a noncharacteristic Lax shock that is

$$
\lambda_{1}\left(u^{-}(t)\right), \cdots, \lambda_{p-1}\left(u^{-}(t)\right)<s^{\prime}(t)<\lambda_{p}\left(u^{-}(t)\right)<\cdots<\lambda_{n}\left(u^{-}(t)\right),
$$

MSC 2000: 35L65,35L67.

Keywords : hyperbolic system of conservation laws, viscous shocks, stability, vanishing viscosity. 


$$
\lambda_{1}\left(u^{+}(t)\right), \cdots, \lambda_{p}\left(u^{+}(t)\right)<s^{\prime}(t)<\lambda_{p+1}\left(u^{+}(t)\right)<\cdots<\lambda_{n}\left(u^{+}(t)\right) .
$$

A general conjecture is that the admissible solutions of (1) can be obtained as limits of solutions of

$$
u_{t}^{\varepsilon}+f\left(u^{\varepsilon}\right)_{x}=\varepsilon u_{x x}^{\varepsilon},
$$

when $\varepsilon$ tends to zero. This conjecture has been proved for scalar conservation laws by using the maximum principle [15] and for some special $2 \times 2$ systems by the method of compensated compactness [3]. This conjecture motivated the work of Goodman and Xin [6], who have shown that $u$ is the limit of a solution of (4) under a smallness assumption on the amplitude of the shock

$$
\sup _{t \in[0, T]}\left|u^{+}(t)-u^{-}(t)\right| \leq \eta_{0},
$$

$\eta_{0}$ being sufficiently small. Their method was based on the construction of an approximate solution of (4) thanks to matched asymptotic expansions techniques and then validation of this expansion thanks to energy estimates. More recently the convergence of $u^{\varepsilon}$ has been proved in [1] for general initial data for (4) having sufficiently small total variation by a completely different method. Here we still focus on the case of a single shock as in [6] and on the matched asymptotic expansion technique but our aim is to remove the smallness assumption(5).

At first let us briefly describe the construction of the approximate solution in [6]. Away from the shock, there is an outer expansion

$$
O(x, t)=u(x, t)+\varepsilon u_{1}(x, t)+\varepsilon^{2} u_{2}(x, t)
$$

where $u$ is the piecewise smooth solution of (1) that we considered, and $u_{i}, i \geq 2$ are solutions of some linear hyperbolic systems. Similarly, near the shock there is an inner expansion

$$
I(x, t)=V(\xi, t)+\varepsilon V_{1}(\xi, t)+\varepsilon^{2} V_{2}(\xi, t)
$$

where $\xi=\frac{x-s(t)}{\varepsilon}+\delta(t)$ is the stretched variable. There is also an expansion of $\delta(t)$ which is a perturbation of the shock position :

$$
\delta(t)=\delta_{0}(t)+\varepsilon \delta_{1}(t) .
$$

The viscous shock profile $V(\xi, t)$ is a solution of

$$
V_{\xi}=f(V)-f\left(u^{-}(t)\right)-s^{\prime}(t)\left(V-u^{-}(t)\right)
$$

such that

$$
\lim _{\xi \rightarrow \pm \infty} V(\xi, t)=u^{ \pm}(t)
$$

The higher order terms are solutions of some linear ordinary differential equations. Taking a smooth function $m$ such that $m(x)=1$ when $|x| \leq 1$ and $m(x)=0$, when $|x| \geq 2$, one finally gets an approximate solution of (4)

$$
u^{a p p}(x, t)=m\left(\frac{x-s(t)}{\varepsilon^{\gamma}}\right) I(x, t)+\left(1-m\left(\frac{x-s(t)}{\varepsilon^{\gamma}}\right)\right) O(x, t)+d(x, t),
$$


where $d$ is an higher order correction term that allows to put the error term in conservative form(see 2)and $\gamma \in\left(\frac{2}{3}, 1\right)$. The approximate solution $u^{a p p}$ then solves

$$
u_{t}^{a p p}+f\left(u^{a p p}\right)_{x}-\varepsilon u_{x x}^{a p p}=R_{x}^{\varepsilon}
$$

where $R^{\varepsilon}=O\left(\varepsilon^{M}\right)$. As explained in [5], it is better to deal with an integrated equation when we study the stability of shocks through energy estimates since for weak shocks it allows to use that $\partial_{x} \lambda_{p}(V)<0$. By choosing the solution of (4) with initial data $u^{a p p}(0, x)$, it is then possible to set $w_{x}=u^{\varepsilon}-u^{a p p}$. $w$ then solves

$$
\begin{aligned}
w_{t}+f^{\prime}\left(u^{a p p}\right) w_{x}-\varepsilon w_{x x}= & Q\left(u^{a p p}, w_{x}\right)+\left(f\left(u^{a p p}\right)-f\left(u^{a p p}-d\right)\right), \\
& w(0, x)=0 .
\end{aligned}
$$

The error term $Q\left(u^{a p p}, w_{x}\right)$ is $\mathcal{O}\left(\left|w_{x}\right|^{2}\right)$. The convergence of $w_{x}$ to zero is shown in [6] by mean of energy estimates. This method leads to the smallness assumption (5). The same kind of result with a more precise description of the convergence and the study of the evolution of an initial layer into a shock layer is shown in [16] by the method of approximate Green's functions of [11], but there is still the restriction(5). This smallness assumption is not sharp even when we study the linear stability of shock profiles. There are strong viscous shocks $V(\xi, \tau)$ which are linearly stable for zero-mass perturbation, that is to say that the solutions of

$$
\begin{gathered}
\partial_{t} u+f^{\prime}(V(\xi, \tau)) \partial_{\xi} u-\partial_{\xi \xi} u=0, \\
u(0, x)=u_{0}(\xi)
\end{gathered}
$$

tend to zero when $t \rightarrow+\infty$. However, when the shock does not satisfy a smallness assumption, the classical energy estimates are not sufficient to prove the stability. The main difficulty is due to the fact that the energy tends to zero when $t \rightarrow+\infty$ but not in a monotonous way. To conclude, refined methods are needed as in [10], [17]. Note that these methods do not apply in our time dependent setting since they both rely on the Laplace transform. The aim of this note is to explain the result of [14] about the convergence of $u^{\varepsilon}$ towards $u^{a p p}$ when the viscous profiles are linearly stable.

We now detail more precisely our hypotheses. At first, we assume that

$(\mathbf{H 0}) \forall t \in[0, T]$, there exists a viscous profile $V(\cdot, t)$ which is a solution of $(8,9)$.

Note that thanks to(2),(3), $u^{+}(t)$ and $u^{-}(t)$ are hyperbolic rest points for the ordinary differential equation (8), consequently, we have for any $\alpha$,

$$
\begin{gathered}
\left|\partial_{t}^{\alpha} V(\xi, t)-\partial_{t}^{\alpha} u^{+}(t)\right| \leq e^{-\omega \xi}, \forall \xi \geq 0,\left|\partial_{t}^{\alpha} V(\xi, t)-\partial_{t}^{\alpha} u^{-}(t)\right| \leq e^{\omega \xi}, \forall \xi \leq 0, \\
\left|\partial_{\xi}^{\alpha} V(\xi, t)\right| \leq e^{-\omega|\xi|}, \forall \xi,
\end{gathered}
$$

for some $\omega>0$.

Now, let us formalize the notion of linear stability. Consider for each $\tau \in[0, T]$, the operator

$$
L_{\tau} v=v_{\xi \xi}-\left(f^{\prime}(V(\xi, \tau))-s^{\prime}(\tau)\right) v_{\xi},
$$

in $L^{p}$ with domain $W^{2, p}$ for $p<+\infty$. For each time $\tau$ we want the profile $V(\xi, \tau)$ to be linearly stable. As stated in [17], it is equivalent to the Evans function criterion 
$(\mathbf{H}) \forall \tau \in\left[0, T^{*}\right], L_{\tau}$ is such that $D_{\tau}(\lambda) \neq 0 \forall \lambda, \Re \lambda \geq 0$,

where $D_{\tau}$ is the Evans function of $L_{\tau}$. We refer to [4] and [17] for a precise definition of the Evans function which is a kind of characteristic polynomial for the operator $L_{\tau}$. This hypothesis means that $L_{\tau}$ does not have eigenvalues in the closed right half plane (the unstable half plane)and that zero is not a generalized eigenvalue(note that zero is in the essential spectrum of $L_{\tau}$, hence this assumption on the Evans function is necessary to overcome this neutral case in stability theory). Indeed this hypothesis is necessary and sufficient for the linear stability of Lax shocks shocks as stated in [17]. Note that $(\mathbf{H})$ can be checked by energy methods in the case of weak shocks. At this stage we see another justification of the fact that it is really better to deal with an "integrated" equation. Actually if we deal with the original linear operator

$$
\tilde{L}_{\tau} v=v_{\xi \xi}-\left(\left(f^{\prime}(V(\xi, \tau))-s^{\prime}(\tau)\right) v\right)_{\xi}
$$

the Evans function vanishes at zero(because of the translational invariance of the problem the derivative $V_{\xi}$ of the viscous profile is in the kernel of $\tilde{L}_{\tau}$ ), this yields a much more complicated behaviour of the Green's function of the evolutionary problem [17]. The main theorem of this note is :

Theorem 1 Assuming (H0) and $(\mathbf{H})$, there exists an high order approximate expansion under the form(10). Moreover, we have

$$
\left\|u^{\varepsilon}-u^{a p p}\right\|_{L^{\infty}\left([0, T], L^{1}(\mathbb{R})\right)} \rightarrow 0
$$

and

$$
\left\|u^{\varepsilon}-u^{a p p}\right\|_{L^{\infty}([0, T] \times \mathbb{R})} \rightarrow 0
$$

when $\varepsilon$ tends to zero. Consequently, we have

$$
\left\|u^{\varepsilon}-u\right\|_{L^{\infty}\left([0, T], L^{1}(\mathbb{R})\right)} \rightarrow 0
$$

and for any $\eta \in(0,1)$

$$
\sup _{0 \leq t \leq T,|x-s(t)| \geq \varepsilon^{\eta}}\left|u^{\varepsilon}(x, t)-u(x, t)\right| \rightarrow 0
$$

when $\varepsilon$ tends to zero.

We can also get a convergence in $L^{\infty}\left([0, T], W^{m, 1}(\mathbb{R})\right)$ for any $m$. Note that this theorem is sharp since $(\mathbf{H})$ is necessary for linear stability of each profile and since we can expect that linear instability implies nonlinear instability as in [2].

We have a convergence result in $L^{1}$ du to the method of Green's function construction as in [7], [14]. More recently, another approach based on symmetrizors construction was developped, it allows to recover convergence in $L^{2}$ and to deal with multidimensional systems [12], [8].

In the first part, we briefly explain how to find an high order approximate solution $u^{a p p}$ under the form(10). Note that the first terms $u$ and $V$ in the expansions(6), (7) which are solutions of nonlinear equations exist by assumption.

In the second part, we give outlines of the proof of the stability result, the complete proof with a generalization to nonclassical shocks is in [14]. 


\section{High order approximate solution}

\subsection{Construction}

We explain how to construct $u_{1}, V_{1}$ and $\delta_{0}$ which are built simulaneously. A similar construction can be done for the other terms. Note that another type of construction based on boundary layers expansions is done in [9]. By the method of matched asymptotic expansion we find that $u_{1}$ is a solution of

$$
u_{1 t}+\left(f^{\prime}(u) u_{1}\right)_{x}=u_{x x}
$$

for $x \neq s^{\prime}(t)$ and that $V_{1}$ is a solution of the ordinary differential equation

$$
V_{1 \xi \xi}=\left(\left(f^{\prime}(V)-s^{\prime}(t)\right) V_{1}\right)_{\xi}+\delta_{0}^{\prime}(t) V_{\xi}+V_{t}
$$

Moreover we want the two solutions to be valid in an intermediate zone. This gives the matching conditions

$$
V_{1}(\xi, t)=u_{1}^{ \pm}(t)+\left(\xi-\delta_{0}\right) \partial_{x} u_{0}^{ \pm}+o(1)
$$

when $\xi \rightarrow \pm \infty$.

As in [6], it is more convenient to deal with bounded solutions, hence we can choose a smooth $D_{1}$ such that $U_{1}=V_{1}-D_{1}$ solves

$$
\begin{gathered}
U_{1}(\xi, t)=u_{1}^{ \pm}(t)-\delta_{0}(t) \partial_{x} u_{0}^{ \pm}(t)+o(1), \xi \rightarrow \pm \infty, \\
U_{1 \xi \xi}-\left(\left(f^{\prime}(V)-s^{\prime}(t)\right) U_{1}\right)_{\xi}=\delta_{0}^{\prime}(t) V_{\xi}+g(\xi, t),
\end{gathered}
$$

where

$$
|g(\xi, t)| \leq C e^{-\alpha|\xi|}
$$

Setting

$$
G(\xi, t)=\int_{0}^{\xi} g(\eta, t) d \eta
$$

we get

$$
U_{1 \xi}-\left(f^{\prime}(V)-s^{\prime}(t)\right) U_{1}=\delta_{0}^{\prime}(t) V+G(\xi, t)+c(t),
$$

where $c(t)$ is an integration constant and $G(\xi, t) \rightarrow G_{ \pm}(t)$ when $\xi \rightarrow \pm \infty$. To solve the coupled systems(17),(12), (15), the method is to show that for every $\left(t, \delta_{0}(t), c(t)\right)$ we can find a solution of (17) which tends to some limits at $\pm \infty$ depending on $\left(t, \delta_{0}(t), c(t)\right)$. Using these limits and the matching conditions (15) we can rewrite (12) as an initial boundary value problem where $u_{1}$ and $\delta_{0}$ are the only unknowns. To solve (17) for each $t$ and $\delta_{0}$, we use that thanks to our assumption $(\mathrm{H})$, the operator

$$
A_{t} v=v^{\prime}-\left(f^{\prime}(V(\xi, t))-s^{\prime}(t)\right) v
$$

defined from $C_{b}^{1}(\mathbb{R})$ to $C_{b}^{0}(\mathbb{R})$ is onto with a one-dimensional kernel. This comes from classical results in ordinary differential equations [13]. Next, we show that 
every bounded solution $U_{1}$ of (17) tends to some limits when $\xi \rightarrow \pm \infty$. After the computation of these limits, we can combine with (15) to get

$$
A^{+}(t) u_{1}^{+}(t)-A^{-}(t) u_{1}^{-}(t)+\frac{d}{d t}\left(\delta_{0}\left(u^{+}-u^{-}\right)\right)=\frac{d}{d t}\left(u^{+}-u^{-}\right)
$$

where $A^{ \pm}(t)=f^{\prime}\left(u^{ \pm}(t)\right)-s^{\prime}(t)$. Since our assumption (H) and more precisely the assumption $D_{t}(0) \neq 0$ implies (see [18]) the Majda-Liu condition

$$
\operatorname{det}\left(r_{1}\left(u^{-}(t)\right), \cdots, r_{p-1}\left(u^{-}(t)\right),\left(u^{+}(t)-u^{-}(t)\right), r_{p+1}\left(u^{+}(t)\right), \cdots, r_{n}\left(u^{+}(t)\right)\right) \neq 0
$$

we can solve(12), (18) with unknown $u_{1}, \delta_{0}$ thanks to classical techniques(method of characteristics).

\subsection{Bounds on the error term}

Since we want to integrate the equation, we need to choose an approximate solution of (4) with an error term in a conservative form. If we choose an approximate solution under the basic form

$$
u^{a p p}(x, t)=m\left(\frac{x-s(t)}{\varepsilon^{\gamma}}\right) I(x, t)+\left(1-m\left(\frac{x-s(t)}{\varepsilon^{\gamma}}\right)\right) O(x, t),
$$

we find that

$$
u_{t}^{a p p}+f\left(u^{a p p}\right)_{x}-\varepsilon u_{x x}^{a p p}=q .
$$

we do not give the precise form of $q$, but we just point out that it is not a derivative. Hence, following the idea of [6], we choose an approximate solution in the form (10) where $d$ is such that

$$
\begin{gathered}
d_{t}-\varepsilon d_{x x}=-q(x, t), \\
d(0, x)=d_{0}(x) .
\end{gathered}
$$

Thanks to this choice, $u^{a p p}$ now solves

$$
u_{t}^{a p p}+\left(f^{\prime}\left(u^{a p p}\right)\right)_{x}-\varepsilon u_{x x}^{a p p}=\left(f\left(u^{a p p}-d\right)-f\left(u^{a p p}\right)\right)_{x},
$$

and we will be able to integrate the equation. Let us set $R^{\varepsilon}(t, z)=f\left(\tilde{u}^{a p p}-\tilde{d}\right)-$ $f\left(\tilde{u}^{a p p}\right)$, which is the error term of the approximate solution in(19). We can show that

Proposition 2 There exists a positive constant $C$ independent of $\varepsilon$ such that for all $\gamma \in\left(\frac{2}{3}, 1\right), t \in[0, T]$

$$
\begin{gathered}
\left\|R^{\varepsilon}(t, \cdot)\right\|_{L^{1}} \leq C \varepsilon^{3 \gamma},\left\|R_{t}^{\varepsilon}(t \cdot)\right\|_{L^{1}} \leq C \varepsilon^{3 \gamma-\frac{1}{2}},\left\|R_{t t}^{\varepsilon}(t, \cdot)\right\|_{L^{1}} \leq C \varepsilon^{3 \gamma-1}, \\
\left\|R_{z}^{\varepsilon}(t, \cdot)\right\|_{L^{1}} \leq C \varepsilon^{3 \gamma-1},\left\|R_{z z}^{\varepsilon}(t, \cdot)\right\|_{L^{1}} \leq \varepsilon^{2 \gamma-\frac{1}{2}}
\end{gathered}
$$




\section{Stability analysis}

Let us go back to(11). After the change of variable $z=x-s(t)+\varepsilon \delta(t)$, we find

$$
\tilde{w}_{t}+\left(f^{\prime}\left(\tilde{u}^{a p p}(z, t)\right)-s^{\prime}(t)+\varepsilon \delta^{\prime}(t)\right) \tilde{w}_{z}-\varepsilon \tilde{w}_{z z}=Q\left(\tilde{u}^{a p p}, \tilde{w}_{z}\right)+R^{\varepsilon},
$$

where $\tilde{u}^{a p p}(z, t)=u^{a p p}(z+s(t)-\varepsilon \delta(t), t), \tilde{d}(z, t)=d(z+s(t)-\varepsilon \delta(t))$ and $\tilde{w}(z, t)=$ $w(z+s(t)-\varepsilon \delta(t), t)$. We note that now the viscous shock is located at $z=0$.

Note that using the Green's function $G$ of the linear parabolic operator

$$
\mathcal{L}^{\varepsilon} w=w_{t}+\left(f^{\prime}\left(\tilde{u}^{a p p}\right)-s^{\prime}(t)+\varepsilon \delta^{\prime}(t)\right) w_{z}-\varepsilon w_{z z},
$$

$\tilde{w}$ can be considered as a solution of the integral equation

$$
\left.\tilde{w}(t, z)=\int_{0}^{t} \int_{\mathbb{R}} G(t, \tau, z, y)\left(R^{\varepsilon}(z, \tau)\right)+Q\left(u^{a p p}, \tilde{w}_{z}\right)\right) d y d \tau .
$$

Consequently, it suffices to obtain good estimates on the Green's function $G^{\varepsilon}$ to get the nonlinear result of Theorem 1by classical continuous induction arguments for parabolic equations. Let us recall that the Green's function $G(t, \tau, x, y)$ is a solution of $\mathcal{L}^{\varepsilon} G=0$ for $t>\tau$ such that

$$
\lim _{t \rightarrow \tau^{+}} G(t, \tau, x, y)=\delta_{y}(x) I_{n},
$$

and $G(t, x, \tau, y)=0, t<\tau$. The difficult part of the Theorem is then to prove

Theorem 3 There exists a Green's function $G(t, \tau, x, y)$ of $\mathcal{L}_{\varepsilon}$ defined for $0 \leq \tau, t \leq$ $T, z, y \in \mathbb{R}$ such that

$$
\sup _{0 \leq \tau \leq T, y} \int_{0}^{T} \int_{\mathbb{R}}|G(t, \tau, z, y)| d z d t+\sqrt{\varepsilon} \sup _{0 \leq \tau \leq T, y} \int_{0}^{T} \int_{\mathbb{R}}\left|\partial_{z} G(t, \tau, z, y)\right| d z d t
$$

is bounded uniformly in $\varepsilon$.

\section{Proof}

We use the iterative construction of the Green's functions given in [7]. We choose an approximate Green's function $G^{a p p}(t, \tau, z, y)$ under the form

$$
G^{a p p}(t, \tau, z, y)=\sum_{k=1}^{N} S_{k}(t, \tau, z, y) \Pi_{k}(\tau, y)
$$

where $S_{k}(t, \tau, z, y)$ are Green's kernels and $\Pi_{k} \in \mathcal{C}^{\infty}\left([0, T] \times \mathbb{R}, \mathcal{L}\left(\mathbb{R}^{n}\right)\right)$ are such that

$$
\left\|\Pi_{k}(t, x) v\right\| \leq C\|v\|, \forall x \geq 0, t \in[0, T], v \in \mathbb{R}^{n}
$$

and

$$
\sum_{k} \Pi_{k}=\mathrm{Id}
$$


For each $S_{k}$, we define the error $R_{k}(t, \tau, z, y)=\mathcal{L}_{\varepsilon} S_{k}$. We then define the matrix of "error interactions" $\mathcal{M}\left(T_{1}, T_{2}\right)=\left(\sigma_{k l}\left(T_{1}, T_{2}\right)\right)_{1 \leq k, l \leq N}$, where

$$
\sigma_{k l}\left(T_{1}, T_{2}\right)=\sup _{T_{1} \leq \tau \leq T_{2}, y \in \operatorname{Supp} \Pi_{l}} \int_{T_{1}}^{T_{2}} \int_{\mathbb{R}}\left|\Pi_{k}(t, z) R_{l}(t, \tau, z, y)\right| d z d t .
$$

This matrix describes how each part of the approximate Green's function is handled at the next step of the iterative method. Theorem 5 of [7] states that to prove (20) it suffices to check that there exists $\eta$ such that $T_{2}-T_{1} \leq \eta$ implies

$$
\lim _{p \rightarrow+\infty} \mathcal{M}^{p}\left(T_{1}, T_{2}\right)=0 .
$$

Let us now introduce some definitions that are necessary for the construction of our approximate Green's function. We use two smooth cut-off functions $\chi^{+}$and $\chi^{-}$such that $\chi^{+}(z)=\left\{\begin{array}{l}0 \text { if } z \leq 1 \\ 1 \text { if } z \geq 2\end{array}\right.$ and $\chi^{-}(z)=\left\{\begin{array}{l}0 \text { if } z \geq-1 \\ 1 \text { if } z \leq-2\end{array}\right.$. We also assume that the cut-off function $m$ already used was under the form $\left(1-\chi^{+}\right)\left(1-\chi^{-}\right)$. We denote by $P^{ \pm}(t, z), D^{ \pm}(t, z)$ matrices such that

$$
\begin{aligned}
& f^{\prime}(u(z+s(t), t))-s^{\prime}(t)=P^{+} D^{+}\left(P^{+}\right)^{-1}, \forall z>0, \\
& f^{\prime}(u(z+s(t), t))-s^{\prime}(t)=P^{-} D^{-}\left(P^{-}\right)^{-1}, \forall z<0,
\end{aligned}
$$

and $D^{ \pm}( \pm z, t)=\operatorname{diag}\left(\lambda_{1}(u(s(t) \pm z, t))-s^{\prime}(t), \cdots, \lambda_{n}(u(s(t) \pm z, t))-s^{\prime}(t)\right)$, if $z>0$. Setting

$$
\lambda_{i}^{+}(z, t)=\left\{\begin{array}{l}
\lambda_{i}(u(z+s(t), t))-s^{\prime}(t) \text { if } z>0 \\
\lambda_{i}(u(s(t)+0, t))-s^{\prime}(t) \text { if } z \leq 0
\end{array}\right.
$$

and

$$
\lambda_{i}^{-}(z, t)=\left\{\begin{array}{l}
\lambda_{i}(u(z+s(t), t))-s^{\prime}(t) \text { if } z<0 \\
\lambda_{i}(u(s(t)-0, t))-s^{\prime}(t) \text { if } z \geq 0
\end{array}\right.
$$

we define the characteristic curves $X_{i}^{ \pm}(t, \tau, y)$ by

$$
\partial_{t} X_{i}^{ \pm}(t, \tau, y)=\lambda_{i}^{ \pm}\left(X_{i}^{ \pm}(t, \tau, y), t\right), t \geq \tau,
$$

with initial data $X_{i}^{ \pm}(\tau, \tau, y)=y$.

We also define the projections along incoming ant outgoing characteristics

$$
\begin{aligned}
\mathcal{P}_{\text {out }}^{+}(t, z) & =P^{+}(t, z) D_{\text {out }}^{+}(t, z)\left(P^{+}\right)^{-1}(t, z), \\
\mathcal{P}_{\text {in }}^{+}(t, z) & =P^{+}(t, z) D_{\text {in }}^{+}(t, z)\left(P^{+}\right)^{-1}(t, z), \\
\mathcal{P}_{\text {out }}^{-}(t, z) & =P^{-}(t, z) D_{\text {out }}^{-}(t, z)\left(P^{-}\right)^{-1}(t, z), \\
\mathcal{P}_{\text {in }}^{-}(t, z) & =P^{-}(t, z) D_{\text {in }}^{-}(t, z)\left(P^{-}\right)^{-1}(t, z),
\end{aligned}
$$

where

$$
\begin{aligned}
D_{\text {out }}^{+} & =\operatorname{diag}(0, \cdots, 0,1, \cdots 1), \text { with } p+1 \text { null coefficients, } \\
D_{\text {in }}^{+} & =\operatorname{diag}(1, \cdots, 1,0, \cdots, 0), \text { with } p \text { unit coefficients, } \\
D_{\text {out }}^{-} & =\operatorname{diag}(1, \cdots, 1,0, \cdots, 0), \text { with } p-1 \text { unit coefficients, } \\
D_{\text {in }}^{-} & =\operatorname{diag}(0, \cdots, 0,1, \cdots, 1), \text { with } p \text { unit coefficients. }
\end{aligned}
$$


Let

$$
G_{T}^{ \pm}(t, \tau, z, y)=\operatorname{diag}\left(\frac{e^{-\frac{\left(z-X_{i}^{ \pm}(t, \tau, y)\right)^{2}}{4 \varepsilon(t-\tau)}}}{\sqrt{4 \pi \varepsilon(t-\tau)}}\right) .
$$

We define Green's functions for the incoming and outgoing waves as

$$
\begin{aligned}
G_{\text {out }}^{+}(t, \tau, z, y) & =\chi^{+}\left(\frac{z}{M_{1} \varepsilon}\right) P^{+}(t, z) D_{\text {out }}^{+}(t, z) G_{T}(t, \tau, z, y)\left(P^{+}(\tau, y)\right)^{-1}=\chi^{+} \tilde{G}_{\text {out }}^{+}, \\
G_{\text {in }}^{+}(t, \tau, z, y) & =\chi^{+}\left(\frac{z}{M_{1} \varepsilon}\right) P^{+}(t, z) D_{\text {in }}^{+}(t, z) G_{T}(t, \tau, z, y)\left(P^{+}(\tau, y)\right)^{-1}=\chi^{+} \tilde{G}_{\text {in }}^{+}, \\
G_{\text {out }}^{-}(t, \tau, z, y) & =\chi^{-}\left(\frac{z}{M_{1} \varepsilon}\right) P^{-}(t, z) D_{\text {out }}^{-}(t, z) G_{T}(t, \tau, z, y)\left(P^{-}(\tau, y)\right)^{-1}=\chi^{-} \tilde{G}_{\text {out }}^{-}, \\
G_{\text {in }}^{-}(t, \tau, z, y) & =\chi^{-}\left(\frac{z}{M_{1} \varepsilon}\right) P^{-}(t, z) D_{\text {in }}^{-}(t, z) G_{T}(t, \tau, z, y)\left(P^{-}(\tau, y)\right)^{-1}=\chi^{-} \tilde{G}_{\text {in }}^{-},
\end{aligned}
$$

where $M_{1}>0$ is to be chosen. We also define

$$
G^{\text {shock }}(t, \tau, z, y)=m\left(\frac{z}{M_{3} \varepsilon}\right) G_{\tau}^{S}(t, \tau, z, y)
$$

where $G_{\tau}^{S}$ is the Green's function of the operator with time frozen in the coefficients

$$
L_{\tau}^{\varepsilon} w=w_{t}+\left(f^{\prime}\left(V\left(\frac{z}{\varepsilon}, \tau\right)\right)-s^{\prime}(\tau)\right) w_{z}-\varepsilon w_{z z} .
$$

Thanks to the stability assumption $(\mathrm{H})$, the analysis of [17] based on Laplace transform applies. Hence, a very precise pointwise behaviour of the Green's function $G_{\tau}^{S}$ is available. Actually we do not use the full expansion of the Green's function given in [17]. We just use that for bounded $y$ the Green's function can be described by Gaussians moving along the outgoing characteristics see [7], [14].

The kernels of the theorem of [7] are $S_{1}=S_{2}=G_{\text {out }}^{-}, S_{3}=G_{\text {in }}^{-}, S_{4}=G^{\text {shock }}$, $S_{5}=G_{i n}^{+}, S_{6}=S_{7}=G_{\text {out }}^{+}$. The truncation functions will be

$$
\begin{aligned}
& \Pi_{1}(\tau, y)=\chi^{-}\left(\frac{y}{M_{2} \varepsilon}\right)\left(1-\chi^{-}\left(\frac{2 y}{M_{3} \varepsilon}\right)\right) \mathcal{P}_{\text {out }}^{-}(\tau, y) \\
& \Pi_{2}(\tau, y)=\chi^{-}\left(\frac{2 y}{M_{3} \varepsilon}\right) \mathcal{P}_{\text {out }}^{-}(\tau, y), \\
& \Pi_{3}(\tau, y)=\chi^{-}\left(\frac{y}{M_{2} \varepsilon}\right) \mathcal{P}_{\text {in }}^{-}(\tau, y), \\
& \Pi_{4}(\tau, y)=m\left(\frac{y}{M_{2} \varepsilon}\right) \\
& \Pi_{5}(\tau, y)=\chi^{+}\left(\frac{y}{M_{2} \varepsilon}\right) \mathcal{P}_{\text {in }}^{+}(\tau, y), \\
& \Pi_{6}(\tau, y)=\chi^{+}\left(\frac{2 y}{M_{3} \varepsilon}\right) \mathcal{P}_{\text {out }}^{+}(\tau, y), \\
& \Pi_{7}(\tau, y)=\chi^{+}\left(\frac{y}{M_{2} \varepsilon}\right)\left(1-\chi^{+}\left(\frac{2 y}{M_{3} \varepsilon}\right)\right) \mathcal{P}_{\text {out }}^{+}(\tau, y) .
\end{aligned}
$$


The constants $M_{1}, M_{2}$ and $M_{3}$ are such that $M_{1} \leq 4 M_{2} \leq 16 M_{3}$ and are carefully choosen at the end of the proof. $G_{1}$ and $G_{5}$ describe the creation and propagation of outgoing waves in a vicinity of the shock layer, $G_{2}$ and $G_{6}$ describe the creation and propagation of outgoing waves away from the shock layer, $G_{3}$ and $G_{7}$ describe the creation and propagation of ingoing waves and $G_{4}$ describes the dynamics of the shock layer. It is important to describe very precisely the propagation of outgoing waves in the vicinity of the shock layer because these waves can interact with the shock layer to give a large but actually very localized error. Note that with this choice we get a relevant approximate Green's function since $G^{a p p}(\tau, \tau, z, y)=\delta_{y}(z) I_{n}$. Moreover $G^{a p p}$ satisifies the estimate (20) hence we can use theorem 5 of [7]. To prove the Theorem, we have to compute $\mathcal{M}$ that is to say the various errors made by the approximate Green's functions $G_{1}, \cdots, G_{8}$. Most of the time, these errors are made by the product of a moving gaussian and an exponentially decreasing factor $e^{-\sigma|z|}$ or a truncation function supported in $\left[M_{i}, 2 M_{i}\right]$. Consequently, a crucial lemma for the estimation of these terms is

Lemma 4 ([7]) Let us define for some trajectory $X(t)$, such that $X(0)=0$,

$$
\begin{gathered}
I(y)=\int_{0}^{+\infty} \int_{0}^{+\infty} \exp \left(-\frac{(x-y-X(t))^{2}}{t}\right) \exp (-\sigma x) \frac{d x d t}{\sqrt{t}} \\
K(y)=\frac{1}{M} \int_{0}^{+\infty} \int_{M}^{2 M} \exp \left(-\frac{(x-y-X(t))^{2}}{t}\right) \frac{d x d t}{\sqrt{t}}
\end{gathered}
$$

i) If $\gamma \geq X^{\prime}(t) \geq \delta>0$, then $I(y)$ is bounded uniformly in $y \geq 0$, and go to 0 as $y \rightarrow+\infty$.

ii) If $-\gamma \leq X^{\prime}(t) \leq-\delta<0$ then $I(y)$ is bounded uniformly in $y \geq 0$.

iii) If $\gamma \geq X^{\prime}(t) \geq \delta>0$ then $K(y)$ is bounded uniformly in $y \geq 0$ and $M$, and goes to 0 as $y-2 M$ goes to $+\infty$.

iv) If $-\gamma \leq X^{\prime}(t) \leq-\delta<0$ then $K(y)$ is bounded uniformly in $y \geq 0$ and $M$.

\section{References}

[1] S. Bianchini and A. Bressan. Vanishing viscosity solutions of nonlinear hyperbolic systems. Preprint, 2001.

[2] B. Desjardins and E. Grenier. Linear instability implies nonlinear instability for various boundary layers. Preprint ENS-Lyon.

[3] R. J. DiPerna. Convergence of the viscosity method for isentropic gas dynamics. Comm. Math. Phys., 91(1):1-30, 1983.

[4] R. A. Gardner and K. Zumbrun. The gap lemma and geometric criteria for instability of viscous shock profiles. Comm. Pure Appl. Math., 51(7):797-855, 1998. 
[5] J. Goodman. Nonlinear asymptotic stability of viscous shock profiles for conservation laws. Arch. Rational Mech. Anal., 95(4):325-344, 1986.

[6] J. Goodman and Z. P. Xin. Viscous limits for piecewise smooth solutions to systems of conservation laws. Arch. Rational Mech. Anal., 121(3):235-265, 1992.

[7] E. Grenier and F. Rousset. Stability of one-dimensional boundary layers by usingGreen's functions. Comm. Pure Appl. Math., 54(11):1343-1385, 2001.

[8] O. Guès, G. Métiver, M. Williams and K. Zumbrun. Multidimensional viscous shocks II : the small viscosity limit. Preprint, 2002

[9] O. Guès, M. Williams. Curved shocks as viscous limits : a boundary problem approach. Preprint, 2001

[10] G. Kreiss and H.-O. Kreiss. Stability of systems of viscous conservation laws. Comm. Pure Appl. Math., 51(11-12):1397-1424, 1998.

[11] T.-P. Liu. Pointwise convergence to shock waves for viscous conservation laws. Comm. Pure Appl. Math., 50(11):1113-1182, 1997.

[12] G. Métivier and K. Zumbrun. Large viscous boundary layers for noncharacteristic nonlinear hyperbolic problems. Preprint, 2002

[13] K. J. Palmer. Exponential dichotomies and transversal homoclinic points. J. Differential Equations, 55(2):225-256, 1984.

[14] F. Rousset. Viscous limits for strong shocks of systems of conservation laws. Preprint, 2001

[15] A. I. Volpert. Spaces BV and quasilinear equations. Mat. Sb.(N.S.), 73(115):255-302, 1967.

[16] S. H. Yu. Zero-dissipation limit of solutions with shocks for systems of hyperbolic conservation laws. Arch. Ration. Mech. Anal., 146(4):275-370, 1999.

[17] K. Zumbrun and P. Howard. Pointwise semigroup methods and stability of viscous shock waves. Indiana Univ. Math. J., 47(3):741-871, 1998.

[18] K. Zumbrun and D. Serre. Viscous and inviscid stability of multidimensional planar shock fronts. Indiana Univ. Math. J., 48(3):937-992, 1999.

U.M.P.A.,

École Normale Supérieure de Lyon,

46 ALlÉE D'ITALIE,

69364 LYON CEDEX 07

frousset@umpa.ens-lyon.fr

www.umpa.ens-lyon.fr $/ \sim$ frousset 